\title{
Applying System Combination to Base Noun Phrase Identification
}

\author{
Erik F. Tjong Kim Sang ${ }^{*}$, Walter Daelemans ${ }^{\alpha}$, Hervé Déjean ${ }^{\tau}$, \\ Rob Koeling ${ }^{\gamma}$, Yuval Krymolowski ${ }^{\beta}$, Vasin Punyakanok ${ }^{l}$, Dan Roth ${ }^{t}$
}

\author{
${ }^{\alpha}$ University of Antwerp) \\ Universiteitsplein 1 \\ B-2610) Wilrijk, Belgium \\ \{erikt,daelem\}@uia.ua.ac.be \\ ${ }^{\beta}$ Bar-Ilan University \\ Ramat Gam, 52900, Isiacl \\ yuvalk(i)macs.biu.ac:il
}

${ }^{\tau}$ Universität Tübingen

Kleine WilholmstraBe 113

D)-72074 Tiubingen, Germany

dojoun@sts.nphil.uni-tucbingen.de

\author{
"University of Illinois \\ 1304 W. Springficld Ave. \\ Urloana, IJ, 61.801, USA \\ \{pmyakan,danr\}@ocs.uiuc.edu
}

\begin{abstract}
Wo use seven machine learning algorithms for one task: identifying base nom phrases. The results have been processed by different system combination mothods and all of these outperformed the best individual result. We have applied the seven learners with the best combinator, a majority vote of the top five systems, to a standard data set and managed to improve the best published result for this data set.
\end{abstract}

\section{Introduction}

Van Halteren et al. (1998) and Brill and Wu (1998) show that part-of-speech tagger performance can be inproved by combining different taggers. By using techniques such as majority voting, crrors made by the minority of the taggers can be removed. Van IIalteren et al. (1998) report that the results of such a combined ap)proach can improve upon the accuracy cror of the best individual system with as much as $19 \%$. The positive effect of system combination for non-language processing tasks has been shown in a large body of machine loarning work.

In this paper we will use system combination for identifying base noun phrases (basoNPs). We will apply seven machine learning algorithms to the same baseNP task. At two points we will apply combination methods. We will start with making the systems process five output representations and combine the resultis by choosing the majority of the output features. Three of the seven systems use this approach. After this we will make an overall combination of the results of the seven systems. There wo will evaluate several system combination meth- ods. The best performing method will be applied to a standard data set for baseNP identification.

\section{Methods and experiments}

In this section we will describe our learning task: recognizing base nom phrases. After this we will describe the data representations we used and the machine loarning algorithms that we will apply to the task. We will conchude with an overview of the combination methods that we will test.

\subsection{Task description}

Base nom phrases (baseNPs) are nom phrases which do not; contain another noun phasase. For (xample, the sentence

$$
\begin{aligned}
& \text { In [ early trading] in [ Hong Kong] } \\
& \text { [ Monday ], [ gold ] was quoted at } \\
& \text { [\$366.50] [ an ounce ] . }
\end{aligned}
$$

contains six bascNl's (marked as phrases between square brackets). 'The phrase $\$ 366.50$ an ounce is a nom phrase as well. However, it is not a bascNP since it contains two other noun phrases. Two baseNl' data sets have been put forward by Ramshaw and Marcus (1995). The main data set consist of four sections of the Wall Street Journal (WSJ) part of the Penn Treebank (Marcus et al., 1993) as training material (sections 15-18, 211727 tokens) and one section as test material (section 20, 47377 tokens) ${ }^{1}$. The data contains words, their part-of-speech

\footnotetext{
${ }^{1}$ This Ramshaw and Marcus (1995) baseNP data set is available via $\mathrm{ft}_{\mathrm{p}} \mathrm{p} / / \mathrm{ftp}$.cis.upenn.edu/pub/chunker/
} 
(POS) tags as computed by the Brill tagger and their baseNP scgmentation as derived from the Treebank (with some modifications).

In the baseNP identification task, performance is measured with three rates. First, with the percentage of detected noun phrases that are correct (precision). Second, with the percentage of noun phrases in the data that were found by the classifier (recall). And third, with the $\mathrm{F}_{\beta=1}$ rate which is equal to $\left(2^{*}\right.$ precision*recall)/(precision+recall). The latter rate has been used as the target for optimization.

\subsection{Data representation}

In our example sentence in section 2.1, noun phrases are represented by bracket structures. It has been shown by Muñoz et al. (1999) that for baseNP recognition, the representation with brackets outperforms other data representations. One classifier can be trained to recognize open brackets $(\mathrm{O})$ and another can handle close brackets (C). Their results can bo combined by making pairs of open and close brackets with large probability scores. We have used this bracket representation $(\mathrm{O}+\mathrm{C})$ as well. However, we have not used the combination stratcgy from Muñoz et al. (1999) but instead used the strategy outlined in Tjong Kim Sang (2000): regard only the shortest possible phrases between candidate open and close brackets as base noun phrases.

An alternative representation for baseNPs has been put forward by Ramshaw and Marcus (1995). They have defined baseNP recognition as a tagging task: words can be inside a baseNP (I) or outside a baseNP (O). In the case that one baseNP immediately follows another baseNP, the first word in the second baseNP reccives tag B. Example:

$$
\begin{aligned}
& \text { In }_{O} \text { early }_{I} \text { trading }_{I} \text { in }_{O} \text { Hong }_{I} \text { Kong }_{I} \\
& \text { Monday }_{B}, O \text { gold }_{I} \text { was }_{O} \text { quoted }_{O} \text { at }_{O} \\
& \$_{I} 366.50_{I} \text { an }_{B} \text { ounce }_{I} \cdot O
\end{aligned}
$$

This set of three tags is sufficient for encoding baseNP structures since these structures are nonrecursive and nonoverlapping.

Tjong Kim Sang (2000) outlines alternative versions of this tagging representation. First, the B tag can be used for the first word of every baseNP (IOB2 representation). Second, instead of the B tag an $\mathrm{E}$ tag can be used to mark the last word of a baseNP immediately before another baseNP (IOE1). And third, the $\mathrm{E}$ tag can be used for every noun phrase final word (IOE2). He used the Ramshaw and Marcus (1995) representation as well (IOB1). We will use these four tagging representations and the $\mathrm{O}+\mathrm{C}$ representation for the system-internal combination experiments.

\subsection{Machine learning algorithms}

This section contains a brief description of the seven machine learning algorithms that we will apply to the baseNP identification task: ALLiS, c5.0, IGTree, MaxEnt, MBL, MBSL and SNoW.

ALLiS $^{2}$ (Architecture for Learning Linguistic Structures) is a learning system which uses theory refincment in order to learn non-recursive NP and VP structures (Déjean, 2000). ALLiS generates a regular expression grammar which describes the phrase structure (NP or VP). This grammar is then used by the CASS parser ( $\mathrm{Ab}$ ney, 1996). Following the principle of theory refinement, the lcarning task is composed of two steps. The first step is the generation of an initial grammar. The generation of this grammar uses the notion of default values and some background knowledge which provides general expectations concerning the imner structure of NPs and VPs. This initial grammar provides an incomplete and/or incorrect analysis of the data. The second step is the refinement of this grammar. During this step, the validity of the rules of the initial grammar is checked and the rules are improved (refined) if necessary. This refinement relies on the use of two operations: the contextualization (in which contexts such a tag always belongs to the phrase) and lcxicalization (use of information about the words and not only about POS).

$\mathrm{C} 5.0^{3}$, a commercial version of $\mathrm{C} 4.5$ (Quinlan, 1993), performs top-down induction of decision trees (TDIDT). On the basis of an instance base of examples, C5.0 constructs a decision tree which compresses the classification information in the instance base by exploiting differences in relative importance of different features. Instances are stored in the tree as paths

\footnotetext{
${ }^{2} \mathrm{~A}$ demo of the NP and VP chunker is available at http://www.sfb441.unitucbingen.de/ dejean/chunker.h tinl

${ }^{3}$ Available from http://www.rulequest.com
} 
of connected nodes ending in leaves which contain classification information. Nodes are connected via arcs denoting feature values. Feature information gain (mutual information between features and class) is used to determine the order in which features are employed as tests at all levels of the tree (Quinlan, 1993). With the full input representation (words and POS tags), wo were not able to run complete experiments. Wo therefore experimented only with the POS tags (with a context of two left and right). We have used the default parameter setting with decision trees combined with value grouping.

Wo have used a nearest neighbor algorithm (IB1-IG, here listed as MBL) and a decision tree algorithm (IGThee) from the 'TiMBL loarning package (Daclemans et al., 1999b). Both algorithms store the training datia and classify new items by choosing the most frecuent classification among training items which are closest to this new item. Jata items are represented as sets of feature-value pairs. Each feature receives a weight, which is based on the amount of information which it provides for computing the classification of the items in the training clata. IB 1-IG uses these weights for computing the distance between a pair of datia items and IGThee uses them for deciding which feature-value decisions should be made in the top nodes of the decision tree (1)aclemans ot al., 1999b). We will use their default parametes except for the IB 1-IG parameter for the number of examined nearest neighbors (k) which we have set to 3 (Daclomans et al., 1999a). The classifiers use a left and right context of four words and partof-speech tags. For the four Jo representations we have used a second processing stage which used a smaller context but which included information about the IO tags predicted by the first processing phase (Tjong Kim Sang, 2000).

When building a classifier, one must gather evidence for predicting the correct class of an item from its context. The Maximum Fntropy (MaxEnt) framework is especially suited for integrating evidence from various information sources. Frequencies of evidence/class combinations (called features) are extracted from a sample corpus and considered to be properties of the classification process. Attention is constirained to models with these properties. The MaxEnt principle now demands that annong all the probability distributions that obey these constraints, the most uniform is chosen. During training, fcatures are assigned wcights in such a way that, given the MaxEnt principle, the training data is matched as woll as possible. During evaluation it is tested which features are active (i.e. a feature is active when the context mects the requirements given by the feature). For every class the weights of the active features are combined and the best scoring class is chosen (Berger ot al., 1996). For the classifier built here the surrounding words, their POS tags and baseNI tags predicted for the previous words are used as evidence. A mixture of simple features (consisting of one of the mentioned information sources) and complex features (combinations thereof) were used. The left context never exceded 3 words, the right context was maximally 2 words. The model was calculated using existing software (Dehaspe, 1997).

MI3SI, (Argannon et al., 1999) uses POS clatia in order to idcntify bascNPs. Inference, relies on a memory which contains all the occurrences of POS secuences which appear: in the beginning, or the end, of a baseNP (in(huding complete phrases). These sequences may include a few context tags, up to a prespecified max_context. During inference, MBSL tries to 'tile' cach POS string with parts of noun-phrases from the memory. If the string could be fully covered by the tiles, it becomes part of a candidate list, ambiguities between candidates are resolved by a constraint propagation algorithm. Adding a context extends the possibilities for tiling, thereby giving more opportunities to better candidates. The approach of MBSL to the problem of identifying baseNPs is sequence-based rather than word-based, that is, decisions are taken per POS sequence, or por candidate, but not for a single word. In addition, the tiling process gives no preference to any direction in the sentence. The tiles may be of any length, up to the maximal length of a phrase in the training data, which gives MBSL a generalization power that compensates for the setup of using only POS tags. The results presented here were obtained by optimizing MBSL parameters based on 5-fold CV on the training data.

SNoW uses the Open/Close model, described in Muñoz et al. (1999). As is shown there, this 


\begin{tabular}{|l|c|c|c|c|c|c|c|c|c|}
\cline { 2 - 10 } \multicolumn{1}{c|}{} & \multicolumn{3}{c|}{ MBL } & \multicolumn{3}{c|}{ MaxEnt } & \multicolumn{3}{c|}{ IGTree } \\
\cline { 2 - 10 } section 21 & $\mathrm{O}$ & $\mathrm{C}$ & $\mathrm{F}_{\beta=1}$ & $\mathrm{O}$ & $\mathrm{C}$ & $\mathrm{F}_{\beta=1}$ & $\mathrm{O}$ & $\mathrm{C}$ & $\mathrm{F}_{\beta=1}$ \\
\hline IOB1 & $97.81 \%$ & $97.97 \%$ & 91.68 & $97.90 \%$ & $98.11 \%$ & 92.43 & $96.62 \%$ & $96.89 \%$ & 87.88 \\
IOB2 & $97.63 \%$ & $97.96 \%$ & 91.79 & $97.81 \%$ & $98.14 \%$ & 92.14 & $97.27 \%$ & $97.30 \%$ & 90.03 \\
IOE1 & $97.80 \%$ & $97.92 \%$ & 91.54 & $97.88 \%$ & $98.12 \%$ & 92.37 & $95.88 \%$ & $96.01 \%$ & 82.80 \\
IOE2 & $97.72 \%$ & $97.94 \%$ & 92.06 & $97.84 \%$ & $98.12 \%$ & 92.13 & $97.19 \%$ & $97.62 \%$ & 89.98 \\
O+C & $97.72 \%$ & $98.04 \%$ & 92.03 & $97.82 \%$ & $98.15 \%$ & 92.26 & $96.89 \%$ & $97.49 \%$ & 89.37 \\
\hline Majority & $98.04 \%$ & $98.20 \%$ & 92.82 & $97.94 \%$ & $\mathbf{9 8 . 2 4 \%}$ & 92.60 & $97.70 \%$ & $97.99 \%$ & 91.92 \\
\hline
\end{tabular}

Table 1: The effects of system-internal combination by using different output representations. A straight-forward majority vote of the output yields better bracket accuracies and $F_{\beta=1}$ rates than any included individual classifier. The bracket accuracies in the columns $\mathrm{O}$ and $\mathrm{C}$ show what percentage of words was correctly classified as baseNP start, baseNP end or neither.

model produced better results than the other paradigm evaluated there, the Inside/Outside paradigm. The Open/Close model consists of two SNoW predictors, one of which predicts the beginning of baseNPs (Open predictor), and the other predicts the end of the phrase (Close predictor). The Open predictor is learned using SNoW (Carlson et al., 1999; Roth, 1998) as a function of features that utilize words and POS tags in the sentence and, given a new sontence, will predict for each word whether it is the first word in the phrase or not. For each Open, the Close predictor is learned using SNoW as a function of features that utilize the words in the sentence, the POS tags and the open prediction. It will predict, for each word, whether it can be the end of the phrase, given the previously predicted Open. Each pair of predicted Open and Close forms a candidate of a bascNP. These candidates may conflict due to overlapping; at this stage, a graph-based constraint satisfaction algorithm that uses the confidence values SNoW associates with its predictions is employed. This algorithm ("the combinator") produces the list of the final baseNPs for each sentence. Details of SNoW, its application in shallow parsing and the combinator's algorithm are in Muñoz et al. (1999).

\subsection{Combination techniques}

At two points in our noun phrase recognition process we will use system combination. We will start with system-internal combination: apply the same learning algorithm to variants of the task and combine the results. The approach we have chosen here is the same as in Tjong
Kim Sang (2000): generate different variants of the task by using different representations of the output (IOB1, IOB2, IOE1, IOE2 and $\mathrm{O}+\mathrm{C})$. The five outputs will converted to the open bracket representation $(\mathrm{O})$ and the close bracket representation $(\mathrm{C})$ and after this, the most frequent of the five analyses of each word will chosen (majority voting, see below). We expect the systems which use this combination phase to perform better than their individual members (Tjong Kim Sang, 2000).

Our seven learners will generate different classifications of the training data and we need to find out which combination techniques are most, appropriate. For the system-external combination experiment, we have evaluated different voting mechanisms, effectively the voting methods as described in Van Halteren et al. (1998). In the first method each classification reccives the same woight and the most frequent classification is chosen (Majority). The second method regards as the weight of each individual classification algorithm its accuracy on some part of the data, the tuning data (TotPrecision). The third voting method computes the precision of each assigned tag per classifier and uses this value as a weight for the classifier in those cases that it chooses the tag (TagPrecision). The fourth method uses both the precision of each assigned tag and the recall of the competing tags (Precision-Recall). Finally, the fifth method uses not only a weight for the current classification but it also computes weights for other possible classifications. The other classifications are determined by examining the tun- 
ing data and registering the correct values for every pair of classifier results (pair-wise voting, sce Van Halteren et al. (1998) for an elaborate explanation).

Apart from these five voting methods we have also processed the output streams with two classifiers: MBL and IGTree. This approach is called classifier stacking. Like Van Halteren et al. (1998), we have used different input versions: one containing only the classifier output and another containing both classifier output and a compressed representation of the data item under consideration. For the latter purpose we have used the part-of-speech tag of the current word.

\section{Results $^{4}$}

We want to find out whether system combination could improve performance of baseNP recognition and, if this is the fact, we want to select the bost combination technique. For this purpose we have performed an experiment with sections 15-18 of the WS.J part of the Penn Treebank as training data (211727 tokens) and section 21 as test data (40039 tokens). Like the data used by Ramshaw and Marcus (1995), this data was retagged by the Brill tagger in order to obtain realistic part-of-speech (POS) tags". The data was segmented into baseNP parts and non-baseNP parts in a similar fashion as the data used by Ramshaw and Marcus (1995). Of the training data, only $90 \%$ was used for training. The remaining $10 \%$ was used as tuning data for determining the weights of the combination techniques.

For three classifiers (MBL, MaxEnt and IGThee) we have used system-internal combination. These learning algorithms have processed five different representations of the out. put (IOB1, IOB2, IOE1, IOE2 and $\mathrm{O}+\mathrm{C}$ ) and the results have been combined with majority voting. The test data results can be found in Table 1. In all cases, the combined results were better than that of the best included system.

The resultis of ALLiS, C5.0, MBSL and SNoW have been converted to the $\mathrm{O}$ and the $\mathrm{C}$ repre-

\footnotetext{
${ }^{4}$ Detailed results of our experiments are available on http://lcg-www.uia.ac.be/ crikt/upcombi/

${ }^{5}$ The retagging was necessary to assure that the performance rates obtained here would be similar to rates obtained for texts for which no Treebank POS tags are available.
}

\begin{tabular}{|l|c|c|c|}
\cline { 2 - 4 } \multicolumn{1}{l|}{ scction 21 } & $\mathrm{O}$ & $\mathrm{C}$ & $\mathrm{F}_{\beta=1}$ \\
\hline Classifier & & & \\
ALLiS & $97.87 \%$ & $98.08 \%$ & 92.15 \\
C5.0 & $97.05 \%$ & $97.76 \%$ & 89.97 \\
IGTrec & $97.70 \%$ & $97.99 \%$ & 91.92 \\
MaxEnt & $97.94 \%$ & $98.24 \%$ & 92.60 \\
MBL & $98.04 \%$ & $98.20 \%$ & 92.82 \\
MBSL & $97.27 \%$ & $97.66 \%$ & 90.71 \\
SNoW & $97.78 \%$ & $97.68 \%$ & 91.87 \\
\hline Simple Voting & & & \\
Majority & $98.08 \%$ & $98.21 \%$ & 92.95 \\
TotPrecision & $98.08 \%$ & $98.21 \%$ & 92.95 \\
TagPrecision & $98.08 \%$ & $98.21 \%$ & 92.95 \\
Precision-Recall & $98.08 \%$ & $98.21 \%$ & 92.95 \\
\hline Pairwise Voting & & & \\
TagPair & $98.13 \%$ & $98.23 \%$ & 93.07 \\
\hline Memory-Based & & & \\
Tags & $98.24 \%$ & $98.35 \%$ & 93.39 \\
Tags + POS & $98.14 \%$ & $98.33 \%$ & 93.24 \\
\hline Decision Trees & & & \\
Tags & $98.24 \%$ & $98.35 \%$ & 93.39 \\
Tags + POS & $98.13 \%$ & $98.32 \%$ & 93.21 \\
\hline
\end{tabular}

Table 2: Bracket accuracies and $F_{\beta=1}$ scorcs for section WSJ 21 of the Penn Trecbank with seven individual classifiers and combinations of them. Each combination performs better than its best individual member. The stacked classifiers without context information perform best.

sentation. Together: with the bracket representations of the other three techniques, this gave us a total of seven O results and seven C results. These two data streams have been combined with the combination techniques described in section 2.4. After this, we built baseNPs from the $\mathrm{O}$ and $\mathrm{C}$ results of each combination technique, like described in section 2.2. 'The bracket accuracies and the $\mathrm{F}_{\beta=1}$ scores for test data can be found in Table 2.

All combinations improve the results of the best individual classifier. The best results were obtained with a memory-based stacked classifier. This is different from the combination results presented in Van Halteren et al. (1998), in which pairwise voting performed best. However, in their later work stacked classifiers outperform voting methods as well (Van Halteren et al., to appear). 


\begin{tabular}{|l|c|c|c|c|}
\cline { 2 - 5 } \multicolumn{1}{c|}{ section 20 } & accuracy & precision & recall & $\overline{\mathrm{F}}_{\beta=1}$ \\
\hline Best-five combination & O:98.32\% C:98.41\% & $94.18 \%$ & $93.55 \%$ & 93.86 \\
\hline Tjong Kim Sang (2000) & O:98.10\% C:98.29\% & $93.63 \%$ & $92.89 \%$ & 93.26 \\
Muñoz et al. (1999) & O:98.1\% C:98.2\% & $92.4 \%$ & $93.1 \%$ & 92.8 \\
Ramshaw and Marcus (1995) & IOB1:97.37\% & $91.80 \%$ & $92.27 \%$ & 92.03 \\
Argamon et al. (1999) & - & $91.6 \%$ & $91.6 \%$ & 91.6 \\
\hline
\end{tabular}

Table 3: The overall performance of the majority voting combination of our best five systems (selected on tuning data performance) applied to the standard data set put forward by Ramshaw and Marcus (1995) together with an overview of earlier work. The accuracy scores indicate how often a word was classified correctly with the representation used $(\mathrm{O}, \mathrm{C}$ or IOB1). The combined system outperforms all earlier reported results for this data set.

Based on an carlier combination study (Tjong Kim Sang, 2000) we had expected the voting methods to do better. We suspect that their performance is below that of the stacked classifiers because the difference between the best and the worst individual system is larger than in our earlicr study. We assume that the voting methods might perform better if they were only applied to the classifiers that perform well on this task. In order to test this hypothesis, we have repeated the combination cxperiments with the best $\mathrm{n}$ classifiers, where n took values from 3 to 6 and the classificrs were ranked based on their performance on the tuning data. The best performances were obtained with five classifiers: $F_{\beta=1}=93.44$ for all five voting methods with the best stacked classificr reaching 93.24. With the top five classificrs, the voting methods outperform the best combination with seven systems ${ }^{6}$. Adding extra classification results to a good combination system should not make overall performance worse so it is clear that there is some room left for improvement of our combination algorithms.

We conclude that the best results in this task can be obtained with the simplest voting method, majority voting, applied to the best five of our classifiers. Our next task was to apply the combination approach to a standard data set so that we could compare our results with other work. For this purpose we have used

\footnotetext{
${ }^{6}$ We are unaware of a good method for determining the significance of $\mathrm{F}_{\beta=1}$ differences but we assume that this $\mathrm{F}_{\beta=1}$ difference is not significant. However, we believe that the fact that more combination methods perform well, shows that it easier to get a good performance out of the best five systems than with all seven.
}

the data put forward by Ramshaw and Marcus (1995). Again, only $90 \%$ of the training data was used for training while the remaining $10 \%$ was reserved for ranking the classifiers. The seven learners wore trained with the same parameters as in the previous experiment. Three of the classifiers (MBL, Maxint and IGTree) used system-internal combination by processing different output representations.

The classifier output was converted to the $O$ and the $\mathrm{C}$ representation. Based on the tuning data performance, the classifiers ALLiS, IGTREE, MaxEnt, M]3I and SNoW were solected for being combined with majority voting. After this, the resulting $\mathrm{O}$ and $\mathrm{C}$ representations were combined to baseNPs by using the method described in section 2.2. The results can be found in Table 3. Our combined system obtains an $\mathrm{F}_{\beta=1}$ score of 93.86 which corresponds to an $8 \%$ error reduction compared with the best published result for this data set (93.26).

\section{Concluding remarks}

In this paper we have examined two methods for combining the results of machine lcarning algorithms for identifying base noun phrases. In the first method, the learner processed different output data representations and the results were combined by majority voting. This approach yielded better results than the best included classifier. In the second combination approach we have combined the results of seven learning systems (ALLiS, C5.0, IGTree, MaxEnt, MBL, MBSL and SNoW). Here we have tested different combination methods. Each combination 
method outperformed the best individual learning algorithm and a majority vote of the top five systems performed best. Wo have applied this approach of system-internal and systemexternal combination to a standard data set for base noun phrase identification and the performance of our system was better than any other published result for this data set.

Our study shows that the combination methods that wo have tested are sensitive for the inclusion of classifier results of poor quality. This leaves room for improvement of our results by evaluating other combinators. Another interesting approach which might lead to a better performance is taking into account more context information, for example by combining complete phrases instead of independent brackets. It would also be worthwhile to evaluate using more elaborate methods for building baseNPs out of open and close bracket candidates.

\section{A.cknowledgements}

Déjcan, Koeling and T.jong Kim Sang are funded by the TMR network Learning Computational Grammars? Promyakanok and Roth are supported by NFS grants IIS-9801638 and SBR9873450 .

\section{References}

Steven Abney. 1996. Partial parsing via finitestate cascades. In In Procectings of the LSSSLLI 'g6 Robust Parsing Workshop.

Shlomo Argannon, Ido Dagan, and Yuval Krymolowski. 1999. $\Lambda$ momory-based approach to learning shallow natural language patterns. Journal of Experimental and Theoretical AI, $11(3)$.

Adam L. Berger, Stephen A. Dellal'ietra, and Vincent J. DellaPietra. 1996. A maximum entropy approach to natural language processing. Computational linguistics, 22(1).

Eric Brill and Jum Wu. 1998. Classificr combination for improved lexical disambiguation. In Proceedings of COLING-ACL '98. Association for Computational Jinguistics.

A. Carlson, C. Cumby, J. Rosen, and D. Roth. 1999. The SNoW learning architecture. Technical Report UIUCDCS-R-992101, UIUC Computer Science Department, May.

\footnotetext{
${ }^{7}$ http: //lck-www.uia.ac.be/
}

Walter Daclemans, Antal van den Bosch, and Jakub Zavrel. 1999a. Forgetting exceptions is harmful in language learning. Machine Learning, 34(1).

Walter Daclemans, Jakub Zavrel, Ko van der Sloot, and Antal van den Bosch. 1999b. TiMBL: Tilburg Memory Based Learner, version 2.0, Reference Guide. ISK Technical Report 99-01. http://ilk.kub.nll/.

Luc Dehaspe. 1997. Maximum entropy modeling with clausal constraints. In Proceedings of the 7th International Workshop on Inductive Logic Programming.

Hervé Déjean. 2000. Theory refinement and natural language processing. In Proceedings of the Colingi2000. Association for Computational Linguisties.

Mitchell P. Marcus, Beatrice Santorini, and Mary Ann Marcinkiewic\%. 1993. Building a large annotated corpus of english: the penn treebank. Computational Linguistics, 19(2).

Marcia Munoz, Vasin P'unyakanok, Jan Roth, and Dav Zimak. 1999. A loarning approach to shallow parsing. In Proceedings of EMNLP-WVLC'99. Association for Computational Linguistics.

J. Ross Quinlan. 1993. c4.5: Programs for $\mathrm{Ma}^{-}$ chine Learning. Morgan Kaufmann.

Lance A. Ramshaw and Mitchell P. Marcus. 1995. Text chunking using transformationbased learning. In Proceedings of the Third ACL Workshop on Very Large Corpora. Association for Computational Linguistics.

D. Roth. 1998. Learning to resolve natural language ambiguities: $\Lambda$ unified approach. In AAAI-98.

Erik F. Tjong Kim Sang. 2000. Noun phrase recognition by system combination. In Proceedings of the ANIP-NAACL-2000. Seattle, Washington, USA. Morgan Kaufman Publishers.

Hans van Halteren, Jakub Zavrel, and Walter Daclemans. 1998. Improving data driven wordclass tagging by system combination. In Proceedings of COLING-ACL '98. Association for Computational Linguistics.

Hans van Halteren, Jakub Zavrel, and Walter Daclemans. to appear. Improving accuracy in nlp through combination of machine learning systems. 